\title{
Références bibliographiques du dossier « dimension économique des politiques éducatives »
}

Marie-France Pamart

\section{OpenEdition}

Journals

Édition électronique

URL : http://journals.openedition.org/ries/3007

DOI : 10.4000/ries.3007

ISSN : 2261-4265

Éditeur

Centre international d'études pédagogiques

Édition imprimée

Date de publication : 1 juin 1999

ISSN : 1254-4590

Référence électronique

Marie-France Pamart, «Références bibliographiques du dossier « dimension économique des politiques éducatives » ", Revue internationale d'éducation de Sèvres [En ligne], 22 | 1999, mis en ligne le 13 mai 2013, consulté le 30 avril 2019. URL : http://journals.openedition.org/ries/3007 ; DOI : $10.4000 /$ ries.3007

Ce document a été généré automatiquement le 30 avril 2019

(C) Tous droits réservés 


\title{
Références bibliographiques du dossier « dimension économique des politiques éducatives »
}

\author{
Marie-France Pamart
}

\section{Généralités}

1 PAUL Jean-Jacques (coord.), Administrer, gérer, évaluer les systèmes éducatifs: une encyclopédie pour aujourd'hui, Paris, ESF, « Pédagogies outils», 1999, $360 \mathrm{p}$.

En s'appuyant sur l'histoire de l'économie de l'éducation, cet ouvrage en présente les enjeux actuels, la dimension historique des dépenses d'éducation, les questions de coût et de financement de l'éducation, d'insertion professionnelle et de rendement de l'éducation.

DELAMOTTE Éric, Une introduction à la pensée économique en éducation, Paris, PUF, "Pédagogie d'aujourd'hui », 1998, $213 \mathrm{p}$.

Cet ouvrage retrace l'histoire de la relation entre l'économie et l'éducation, et s'interroge sur le processus selon lequel le tout économique s'est mis en place dans le monde éducatif des pays industrialisés; il explore aussi les conséquences de ce phénomène auprès des enseignants; ceux-ci éprouvent les plus grandes difficultés à le percevoir et à détecter que ce dernier intervient maintenant directement dans leur travail proprement pédagogique. Il ne s'agit pas ici d'opposer "l'homo œconomicus à l'homo academicus » mais bien, au contraire, d'analyser les liens qui se sont tissés entre eux et la réciprocité des facteurs de transformation de l'économique et de l'éducatif.

3 L'éducation, l'État et le local, Éducation et sociétés, $n^{\circ} 1,1998$.

Cette analyse des politiques éducatives tente de poser la problématique de redéfinition du partage des compétences et des responsabilités entre les échelons national, régional et local des systèmes éducatifs à la suite des processus de décentralisation et de déconcentration qui ont affecté ceux-ci. Un chapitre étudie particulièrement la notion de performance appliquée au secteur éducatif et d'utilité d'indicateurs de comparaison. 
CAREIL Yves, De l'école publique à l'école libérale: sociologie d'un changement, Rennes, Presses universitaires de Rennes, «Le sens social», 1998, 245 p.

On trouvera dans cet ouvrage une analyse des mécanismes sous-jacents à la transformation de l'école laïque et publique en une école libérale. Les changements sociaux, la mondialisation du marché, l'ébranlement de la condition salariale, la subordination du politique à l'économique, tout cela contribue au démantèlement du service public et laïque d'éducation et à l'instauration progressive d'une école à plusieurs vitesses où le rôle consumériste de certains parents et leur "professionnalisation » accélèrent le processus.

MINGAT Alain, TAN Jee-Peng, The Mechanics of Progress in Education: Evidence from CrossCountry Data, Dijon, IREDU (Document de travail), 1998/09, $46 \mathrm{p}$.

6 Peut-on mesurer les bénéfices de l'investissement dans les ressources humaines?, Dossier Formation professionnelle, $n^{\circ} 14,1998 / 08,92 p$.

7 HEYNEMAN Stephen-Paul, L'économie de l'éducation: désillusions et espoirs, Perspectives (Unesco), Vol. XXV, $n^{\circ} 4$, 1995/12, p. 611-642, bibliogr.

L'auteur propose un «code de conduite» pour mieux adapter la recherche en économie de l'éducation aux besoins de la communauté éducative.

8 CORREA Hector, The Microeconomic Theory of Education, International Journal of Educational Research, vol. 23, n' 5,1995/07, p. 405-471, bibliogr.

9 CARNOY Martin (éd.), International Encyclopedia of Economics Education, Oxford, Pergamon Press, 1995, $2^{2} e ́ d ., 494 p$.

10 Économie de l'éducation, Économie \& Prévision, n¹16, 1994/12, p. 1-178.

La matière de ce numéro est constituée principalement par les contributions de l'IREDU et de l'AFSE (Association française de science économique) à l'occasion d'un colloque consacré à « l'économie des ressources humaines » réuni à Dijon les 27 et 28 mai 1993 ; ces contributions tournent autour de la question de l'efficacité de l'enseignement qui devient de plus en plus un enjeu proprement économique, efficacité interne qui conduit à s'interroger sur les déterminants des résultats scolaires, efficacité externe qui conduit à s'interroger sur les liens entre l'éducation et la qualité du capital humain à travers ses effets sur la productivité, la rémunération, le rendement de l'investissement en formation.

11 GRAVOT Pierre, Économie de l'éducation, Paris, Économica, 1993, 244 p., bibliogr.

A la fois théorique et concrète, cette analyse synthétique de l'économie de l'éducation s'articule autour de quatre grands thèmes: la demande d'éducation, l'offre d'éducation, l'impact de l'éducation sur l'emploi et l'économie, et enfin la mise en œuvre de la politique éducative.

12 ODDEN Allan (coord.), Symposium: Micro-Approaches to Educational Productivity, Educational Evaluation and Policy Analysis, vol. 14, $n^{\circ} 4,1992, p .303-376$, bibliogr.

BOUMAHDI Rachid, Les rendements de l'éducation: analyse et problèmes économétriques, Thèse pour le doctorat en sciences sociales soutenue le 6 décembre 1991, Toulouse, Université des sciences sociales, 1992.

Cette thèse analyse quelques problèmes et difficultés économétriques qui surgissent dans la mesure des rendements de l'éducation. Le caractère endogène de l'éducation est affirmé en utilisant le test d'exogénéité de Hausman. L'évolution de la rentabilité des études a été calculée. 
14 JAROUSSE Jean-Pierre, L'économie de l'éducation: du "capital humain» à l'évaluation des processus et des systèmes éducatifs, Perspectives documentaires en éducation, $n^{\circ} 23,1991, p$. 79-105, bibliogr.

L'économie de l'éducation est multiple dans son appréhension des différentes dimensions du phénomène; après un historique sur la naissance de la notion de capital humain et une étude $d u$ fonctionnement $d u$ marché du travail, cet article s'attache plus particulièrement à étudier les divers aspects de cette discipline, de l'analyse de la demande d'éducation à l'évaluation du fonctionnement des systèmes éducatifs, du rapport entre l'efficacité externe des systèmes d'enseignement et leur efficience économique.

KRAFT Richard H., NAKIB Yasser, The "New" Economics of Education: Towards a «Unified" Macro/Micro-Educational Planning Policy, Revue internationale de pédagogie, vol 37, $n^{\circ} 3,1991$ p. 299-317.

Education Counts: an Indicator System to Monitor the Nation's Educational Health: Special Study Panel on Education Indicators, Washington, Department of education, 1991, $119 \mathrm{p}$.

COHN Elchanan, GESKE Terry G., The Economics Of Education, Oxford, Pergamon Press, 1990, $3^{e}$ éd., XVIII-430 p., bibliogr.

18 GUTHRIE James W, The Evolving Political Economy of Education and the Implications for Educational Evaluation, Educational Review, vol. 42, n² 2, 1990, p. 109-131, bibliogr.

19 EASTON Peter, KLEES Steven, Éducation et économie : autres perspectives, Perspectives, vol. XX-4, $n^{\circ} 76,1990, p .457-474$.

Après un bref rappel historique du développement de la pensée économique, l'article analyse la théorie du capital humain dans l'économie de l'éducation puis passe en revue les «approches de l'éducation et de l'économie propres à l'économie politique institutionnaliste et radicale »; il s'achève sur quelques suggestions pour repenser l'articulation entre l'économie et l'éducation.

EICHER Jean-Claude, Économie de l'éducation, Encyclopédie économique, Paris, Económica, 1990.

\section{Points de vue des instances internationales et évaluation}

21 POWER Colin N., HUSEN Torsten, BHOLA H.S., BURNETT Nicholas, PATRINOS Harry Antony, MCGINN Noël P., Le rapport Delors et le document de la Banque mondiale sur l'éducation, Perspectives, vol. XXVII, $n^{\circ}$ 2, 1997/06, p. 197-263, bibliogr.

Les deux rapports les plus importants parus au sujet de l'éducation à un niveau international sont le rapport Delors soumis à l'UNESCO et le document du secteur éducation de la Banque mondiale. Les objectifs et les méthodes adoptés pour élaborer ces deux documents sont très différents. Les analyser ensemble peut s'avérer utile pour mesurer les possibilités et les limites des conceptions internationales de l'éducation.

PEANO Serge, Unesco, Commission internationale sur l'éducation pour le XXI siècle, Le financement des systèmes éducatifs, Paris, Unesco, EDC/I/2, 1993/02, 9 p.

Rapport annuel 1997, Washington, Banque mondiale, 1997, $271 \mathrm{p}$.

Ce présent rapport couvre la période qui va du $1^{\mathrm{er}}$ juillet 1996 au 30 juin 1997. Après un bref exposé sur ce que représente la Banque mondiale et son groupe, composé de cinq institutions dont les deux principales sont la Banque internationale pour la 
reconstruction et le développement (BIRD) et l'Association internationale de développement (IDA), et une vue d'ensemble de ses activités pendant l'exercice 97, il passe en revue les principaux programmes de lutte entrepris contre la pauvreté.

BRAY Mark, Decentralization of Education: Community Financing, Washington, Banque mondiale, Directions in Development, 1996/12, VIII-51 p.

Priorités et stratégies pour l'éducation: une étude de la Banque mondiale, Washington, Banque mondiale, 1995, XVI-195 p., bibliogr.

Dans les pays en développement, l'éducation a progressé, mais il est encore nécessaire d'en élargir l'accès dans certains cas, d'en améliorer l'équité et la qualité et, éventuellement, d'en accélérer la réforme. En ce qui concerne le financement, l'éducation de base doit être privilégiée dans les dépenses publiques. Il est aussi nécessaire de trouver de nouvelles sources de fonds et de réaliser des gains d'efficacité permettant des économies. Tout cela conduit, dans la perspective d'une participation accrue des familles et d'une autonomie grandissante des établissements, à une redéfinition du rôle de l'État pour faciliter la mise en œuvre du changement.

26 La Banque mondiale et l'éducation, Washington, Banque mondiale, 1994, 16 p.

Politique; efficacité des investissements éducatifs; maîtrise des coûts unitaires ; diversification des sources de financement.

Regards sur l'éducation 1998 : les indicateurs de l'OCDE, Paris, OCDE, 1998, 468 p.

Cette édition 1998 fournit une batterie d'indicateurs encore plus riche que les précédentes pour apprécier plus finement encore l'état actuel de l'éducation à l'échelle internationale. Les différents chapitres abordent successivement: le contexte démographique, économique et social ; les ressources financières et humaines investies par les pays dans l'éducation; l'accès à l'éducation et les taux de réussite ; l'activité professionnelle des jeunes âgés de 15 à 29 ans; l'environnement pédagogique et l'organisation scolaire. Enfin, le dernier chapitre présente des indicateurs ayant trait aux résultats des élèves et à leur devenir social et professionnel.

HEALY TOM (coord.), ISTANCE David, L'investissement dans le capital humain: une comparaison internationale, Paris, OCDE, 1998, $121 \mathrm{p}$.

«Capital humain: connaissances, qualifications, compétences et autres qualités possédées par un individu et intéressant l'activité économique "; c'est dans cette acception que les pays de l'OCDE ont débattu du sujet en privilégiant les stratégies mises en œuvre pour promouvoir prospérité économique, plein emploi et cohésion sociale. L'objectif de ce rapport est de clarifier ce que l'on sait du capital humain et de sa mesure alors que les conceptions ont évolué sur les éléments qui le constituent, sur son caractère hétérogène et sur le type de formation qui répondra le mieux aux objectifs des politiques publiques.

Analyse des politiques éducatives 1997, Paris, OCDE, «Indicateurs des systèmes d'enseignement », 1997, $116 p$.

Complément indispensable de « Regards sur l'éducation : les indicateurs de l'OCDE », cet ouvrage approfondit les questions actuelles de politique en s'appuyant sur une sélection d'indicateurs. Les sujets abordés sont: les coûts et les dépenses, le capital humain disponible dans les pays de l'OCDE, les performances en " littératie », l'échec et la réussite scolaire, l'efficacité de l'enseignement supérieur face aux nouveaux intérêts et besoins des étudiants. 
MILLER Riel (rap.), Mesurer le capital humain : vers une comptabilité du savoir acquis, Paris, OCDE, 1996, $126 \mathrm{p}$.

Fruit d'une collaboration entre le Comité de l'éducation et le Comité de l'emploi, du travail et des affaires sociales de l'OCDE, le rapport a comme objet d'étude les investissements dans la formation continue et permanente. Il vise à cerner le lien entre le capital humain et les performances économiques, à repenser les systèmes d'information et de prise de décision en matière de ressources humaines. L'analyse fait appel à la recherche effectuée dans trois disciplines : l'économie, la comptabilité et l'éducation.

31 Évaluer et réformer les systèmes éducatifs, Paris, OCDE, 1996, 96 p.

L'étude, exprimant différents points de vue d'experts, aborde plusieurs aspects de l'évaluation dans l'enseignement: rôle dans les contextes d'élaboration de la politique économique des pays membres de l'OCDE, fonctions dans le système éducatif. Elle propose un ensemble de critères afin de permettre aux responsables politiques et autres décideurs de concevoir des stratégies adaptées à chaque situation.

32 LADERRIÈRE Pierre, Les examens de politiques nationales d'éducation à l'OCDE, Sciences de l'éducation pour l'ère nouvelle (les), $n^{\circ} 2-3,1995 / 06$.

L'auteur présente ici les différentes étapes de la procédure dite d'« examen des politiques nationales d'éducation" conduite par l'OCDE; il indique également la méthodologie suivie, ainsi que les résultats, les mesures recommandées et le suivi.

LOWE John (coord.), Les normes de résultats dans l'enseignement: à la recherche de la qualité, Paris, OCDE, 1995, $239 \mathrm{p}$.

L'ouvrage présente la synthèse de dix études de cas par pays sur les normes de l'évaluation. Chaque analyse rappelle le contexte éducatif, le processus d'élaboration des normes, l'évolution, le contenu et la mise en œuvre des normes de résultats, les problèmes et les aspects essentiels des expériences réalisées.

RUMBERGER Russel W., Les résultats économiques en tant qu'indicateur des résultats de l'enseignement, in : Évaluer l'enseignement ; de l'utilité des indicateurs internationaux, Paris, OCDE, 1994, p. 287-309, bibliogr.

Dans ce livre consacré aux indicateurs de l'enseignement, un chapitre est plus particulièrement dédié à l'interaction entre l'économique et l'éducatif; l'analyse aboutit à la conclusion que les indicateurs économiques peuvent être utiles pour évaluer les résultats du système d'enseignement mais qu'il est, d'autre part, très difficile de déterminer avec précision dans quelle mesure les produits de l'enseignement, plutôt que d'autres facteurs, contribuent aux résultats économiques et sociaux.

LAPORTE Bruno, Financing Education and Training in Central and Eastern Europe: a New

Social Contract, Conférence on Education and the Economy in Central and Eastern Europe 29th june/1st july 1992, Paris, OECD, 1992, 16 .

GARNIER Michel (dit.), L'état de l'école $n^{\circ} 8: 30$ indicateurs sur le système éducatif français Paris, Ministère de l'Éducation nationale de la Recherche et de la Technologie, 1998/10, 78 p.

Cette huitième édition de l'état de l'école poursuit l'effort d'information régulier, entrepris depuis 1991, sur l'état et l'évolution de notre système éducatif, de la maternelle à l'enseignement supérieur, sans oublier la formation continue. Cet état des lieux repose sur un ensemble relativement stable d'indicateurs touchant au coût du système, à son activité et à ses résultats.

37 THÉLOT Claude, L'évaluation du système éducatif, Paris, Nathan, "Fac. Éducation», 1993, 160 p., bibliogr. 
L'ouvrage est consacré à l'évaluation d'un état et non d'une politique. L'auteur, après avoir schématiquement rappelé les objectifs et décrit l'organisation du système éducatif, développe les paradigmes de l'évaluation et les principes méthodologiques. L'évaluation du système doit répondre à l'exigence de "rendement », celle de l'établissement scolaire susciter l'émulation. Encore embryonnaire, l'évaluation de la classe est un enjeu important dans la lutte contre l'échec scolaire. À cet acteur essentiel qu'est l'enseignant, le dispositif doit fournir les moyens formalisés de se situer par rapport aux objectifs généraux de l'école et du projet d'établissement lui permettre d'infléchir sapratique pédagogique. Il faut promouvoir une véritable «culture de l'évaluation » afin d'améliorer la qualité de l'enseignement.

\section{Études comparatives et analyses de cas}

Excellence in Education: Views on Improving American Education, Proceedings of a Conference held at the Federal Reserve Bank of New York in November 1997, Economic Policy Review, vol. 4, $n^{\circ} 1$, 1998/03, 129 p., tabl, bibliogr., résumés en anglais.

Actes de la conférence organisée par la Banque fédérale de New York en novembre 1997 sur l'évaluation du système éducatif américain: les communications ont porté sur l'économie de l'éducation (évaluation des dépenses engagées et des résultats), le choix parental et la concurrence entre l'enseignement public et l'enseignement privé, l'évaluation de la qualité des écoles publiques ou privées, la performance de l'enseignement supérieur et de l'accès des étudiants à l'emploi. Les experts réunis en table ronde finale notent les diverses initiatives entreprises pour résoudre le problème majeur du système éducatif aux États-Unis : la dégradation de l'enseignement et des écoles dans les centres-villes et les quartiers pauvres.

CATTERALL James s., Special Section: Economic Analysis of Education Policy, Educational Evaluation and Policy Analysis, vol. 19, $n^{\circ}$ 4, 1997/12, p. 297-372.

ORIVEL François, The Economics of Education: Incentives, Control of Costs, Allocation of Resources, New Actors, Comparative and International Education Society, 40th Annual Meeting, March 6-10, 1996, Williamsburg (USA), Session: Problems and Prospects in European Education, 1996/03, 24 p., bibliogr.

41 DIEBOLT Claude, Éducation et croissance économique : le cas de l'Allemagne au XIX ${ }^{e}$ et $\mathrm{XX}^{e}$ Siècles, Paris, Harmattan, "Bibliothèque de l'éducation », 1995, 178 p.

Ce livre s'adresse à ceux qui cherchent à comprendre les relations entre éducation et croissance économique et ceci à l'aide d'une étude de cas : l'Allemagne. Après un premier chapitre où sont abordés des points de repères théoriques et méthodologiques de la pensée économique, le second étudie les circonstances historiques de la fondation du système éducatif allemand et ses transformations ; dans le troisième chapitre, l'auteur se consacre à l'analyse de ce système éducatif et de son lien avec le développement économique.

LASSIBILLE Gérard, La valorisation des investissements éducatifs par le marché : évidence empirique dans le contexte de l'Espagne, Conférence sur "Éducation et développement économique dans le Monde arabe, en Turquie et en Europe », Fondation Konrad Adenauer, Tunis, 1-5 novembre 1995, 1995/11, 26 .

MINGAT A., Asian Development Bank, Towards Improving our Understanding of the Strategy of High, Performing Asian Economics in the Education Sector, Conference on 
Financing Human Resources in Asia; the Case of High Performing Asian Economies; ADB Headquarters, Manilla, Philippines, 17-18 november 1995, Dijon, IREDU, 1995, 53 p.

L'investissement éducatif et son efficacité : résumés des recherches sélectionnées par appel d'offres lancé par la direction de l'Évaluation et de la Prospective, Éducation et formations : les dossiers, $n^{\circ} 47,1994 / 12$, p. 1-238

L'appel d'offres lancé en 1991 sur ce thème a favorisé des recherches qui se sont déroulées en 1992 et 1993 et dont le bilan a été fait en 1994. Les résumés des quinze propositions de recherche retenues par le comité de sélection sont publiées dans ce dossier. Ils sont regroupés sous trois thèmes : efficacité macro-économique par rapport à la croissance et à la productivité des entreprises ; efficacité économique de la formation de la main-d'œuvre ; efficacité « interne » quant aux compétences et aux savoirs acquis.

ILON Lynn, REIMERS Fernando, PAULINO Amable, GROOTAERT Christian, Structural Adjustment and Education, International Journal of Educational Development, vol. 14, $n^{\circ} 2$, 1994/04, p. 95-142, bibliogr.

PERETTI Claudine, MEURET Denis, L'efficacité de l'Investissement formation, Éducation et formations, $n^{\circ} 34,1993 / 04$, p. 35-41

En France, depuis 1980, la dépense de l'investissement dans la formation initiale n'a cessé de croître. Un groupe de travail dans le cadre du XI ${ }^{\mathrm{e}}$ Plan, s'est interrogé sur l'efficacité de cet investissement et en a conclu que le système éducatif français doit être tenu pour efficace.

HUSEN Torsten (éd.), TUIJNMAN Albert C. (éd.), HALLS W. D., Academia Europaea/s.l. Schooling in Modem European Society. A report of the Academia Europaea, Oxford, Pergamon Press, 1992, 392 p., bibliogr. WOOCK Roger, The "New International Order" in: Educational Research : l'évaluation des formations: points de vue comparatistes, $X V^{e}$ congrès de la CESE, Dijon, 27-06/02-07 1992, atelier 4, Dijon, IREDU, 1992, $10 \mathrm{p}$. MORELAND Neil, HORSBURGH Rod, Auditing: a Tool for Institutional Development, Vocational Aspect of Education (The), vol. 44, $n^{\circ} 1,1992$, p. 29-42, bibliogr.

Education Counts: an Indicator System to Monitor the Nation's Educational Health: Special Study Panel on Education Indicators, Washington, Department of Education, 1991, $119 p$.

\section{Financements : coûts et contrôles}

51 Le financement de la formation professionnelle : approches politiques, Formation professionnelle, $n^{\circ} 13,1998 / 04$, p. 1-99.

Les articles de ce numéro soulignent, mais avec des nuances diverses, plusieurs caractéristiques actuelles concernant l'économie de la formation professionnelle : il y a une tendance forte des pouvoirs publics à introduire les principes du marché dans le domaine de la formation professionnelle ; entre la demande et l'offre de qualifications de la main-d'œuvre, le fossé s'élargit encore ; la situation actuelle et les prévisions dénotent une pénurie croissante de ressources utilisables pour la formation professionnelle; les petites entreprises paraissent relativement incapables d'exploiter les options de formation et d'enseignements professionnels ; des exemples précis et des études de cas, dans un certain nombre de pays, viennent étayer les propos. 


\section{vol. 10, $n^{\circ}$ 2, 1998/06, p. 1-13.}

De nombreux obstacles entravent l'établissement de partenariats égaux entre organismes de développement et pays bénéficiaires. Cependant des efforts réels pour contrer ces difficultés existent. Au niveau de l'Union européenne a été élaboré un projet de « code de conduite " à l'intention des organismes de financement du secteur éducatif. En Suède, l'étude «Partenariat avec l'Afrique » dresse une liste de propositions pour une nouvelle politique envers l'Afrique.

53 Special issue: Perspectives on the Financing of Lifelong Learning, Education Economics, vol. 6, $n^{\circ}$ 3, 1998/12, p. 197-346.

Ce numéro analyse les différents aspects du financement de la formation tout au long de la vie. Comment trouver un système de financement permanent et fiable? Est étudié également le cas où le gouvernement intervient dans le marché de la formation continue ; dans les troisième et quatrième contributions, avec l'aide de plusieurs études menées par l'OCDE, la question est posée de savoir quel bénéfice on peut attendre d'un tel investissement dans la politique de formation continue.

MEHROTRA Santosh, DELAMONICA Enrique, Household Costs and Public Expenditure on Primary Education in Five Low Income Counties: a Comparative Analysis, International Journal of Educational Development, vol. 18, $n^{\circ} 1,1998 / 01$ p. 41-61, tabl., bibliogr.

Résultats de l'analyse demandée par l'UNICEF entre 1993 et 1995 sur le coût de l'enseignement primaire dans cinq pays en développement : le Burkina Faso, le Bhoutan, le Myanmar, l'Ouganda et le Viet Nam.

55 MESNARD Odile, RAGOUCY Christine, BERREUR Christian, Le compte de l'éducation et le compte de l'éducation supérieure: années 1993 à 1996, Éducation et formations: les dossiers, $n^{\circ}$ 93, 1997/10, 175 p., tabl.

Le compte de l'éducation rassemble les données statistiques et comptables relatives aux dépenses d'éducation, à leur financement et aux coûts de production des activités du domaine.

56 Le financement de l'enseignement supérieur, Gestion de l'enseignement supérieur, vol. 8, $n^{\circ} 1$, 1996/03, p. 7-145, bibliogr.

En ce qui concerne structure et gestion financières, une évolution se dessine sous l'effet de divers facteurs. Les articles de ce numéro examinent, à partir des rapports communiqués par le Danemark, la Finlande, la Norvège, les Pays-Bas, la République tchèque et le Royaume-Uni, les questions auxquelles sont confrontés universités et gouvernements des pays occidentaux.

CONSEIL SUPÉRIEUR DE L'ÉDUCATION, Le financement des universités, Avis à la ministre de l'Éducation, Conseil supérieur de l'éducation, Sainte-Foy, 1996,138 p.

58 WEST Anne, WEST Robert, PENNELL Hazel, The Financing of School-Based Education: Changing the Additional Educational Needs Allowance, Education Economics, vol. 3, nº 3, 1995/12, p. 265-275, bibliogr.

59 URWICK James, The Public Costs of Primary Schools: Case Studies from Nigeria, Compare: a Journal of Comparative Education, vol. 23, n 1, 1993/03, p. 71-84, bibliogr.

60 ASSIE-LUMUMBA N'Dri Thérèse, Coûts, financement de l'éducation de base et participation des familles et communautés rurales dans les pays du Tiers-Monde, Paris, IIPE, Problématiques et méthodologies du développement de l'éducation, 1993, 34 p., bibliogr.

Revue internationale d'éducation de Sèvres, 22 | 1999 
61 es coûts des établissements de l'enseignement supérieur, Les Coûts de l'enseignement dans le supérieur: méthodologie d'évaluation et d'analyse, Grenoble, PUG, $1993,48 \mathrm{p}$.

GEORGE Jacques, Les coûts de l'école, Cahiers pédagogiques, n³04-305, 1992/06, p. 11-62

TAN Jee-Peng, MINGAT Alain, Education in Asia: a Comparative Study of Cost and Financing, Washington, World Bank regional and sectoral studies, 1992, 204 p.

PERROT Jean, Les dépenses publiques pour l'enseignement universitaire et le taux de rendement fiscal : le cas de la France, Revue économique, n 1, 1991/01, p. 111-132, graph.

CROSNIER Dominique, PEANO Serge, Le compte de l'éducation : principes et méthodes, Éducation et formations : les dossiers, $n^{\circ} 7,1990 / 10, p .7-110$.

HEYNEMAN Stephen-Paul, Economic Crisis and the Quality of Education, International Journal of Educational Development, vol. 10, $n^{\circ}$ 2-3,1990, p. 115-129.

Meeting Basic Learning Needs: a Vision for the 1990s, Jomtien, World Conference on Education for All, 5-9 March 1991, Background document.

Le financement de l'enseignement supérieur. Tendances actuelles, Paris, OCDE, 1990, 108 p., bibliogr., graph.

\section{RÉSUMÉS}

Cette bibliographie propose une sélection de documents récents (postérieurs à 1990), français et étrangers, référencés dans la base de données du service de documentation du CIEP. Elle est organisée, chronologiquement, autour de quatre axes : généralités sur la pensée économique en éducation et la théorie du capital humain, point de vue des organisations internationales et évaluation, quelques études de cas, financement du système éducatif et contrôle des coûts.

\section{AUTEUR}

\section{MARIE-FRANCE PAMART}

Documentaliste au CIEP. 